\title{
A Textbook Answer to the Debate About Medicare's Prospective Payment System Exemption?
}

\author{
Brian C. Brajcich, MD, and Ryan P. Merkow, MD, MS \\ Department of Surgery, Surgical Outcomes and Quality Improvement Center (SOQIC), Feinberg School of Medicine, \\ Northwestern University, Chicago, IL
}

Most hospitals in the United States are reimbursed by Medicare according to a Prospective Payment System (PPS) model in which payments are calculated based on pre-identified groups of services [i.e., diagnosis-related groups (DRGs)]. When Congress established the PPS in 1983, the law specified exemptions for certain groups of hospitals. One such group included a select number of designated cancer centers, referenced as PPS-exempt cancer centers (PCHs). Over the years, this number increased as several additional cancer centers were written into federal law. ${ }^{1,2}$ The 11 centers that currently possess this status do not abide by standard PPS reimbursement or qualityreporting practices for patients covered by Medicare. ${ }^{3}$ Recently, the role of the PPS exemption policy has been called into question, most notably by the Government Accountability Office (GAO). ${ }^{4}$ The GAO noted that as of 2012, the complexity of patients treated at PCHs, as measured by the average Medicare risk score, the health status of Medicare beneficiaries, and the relative case mix, did not differ from that at PPS teaching hospitals. Additionally, Medicare payments at PCHs were substantially higher, estimated to be at least 0.5 billion dollars per year more than would occur under the PPS. Based on these observations, the GAO concluded that the current PPS exemption policy does not encourage cost saving and recommended that modification or cessation of the policy be considered to incentivize efficiency.

(C) Society of Surgical Oncology 2020

First Received: 17 March 2020;

Published Online: 7 April 2020

R. P. Merkow, MD, MS

e-mail: Ryan.Merkow@nm.org
Several studies have explored whether the quality of care provided by PCHs differs and have reported mixed results. $^{35,6}$ Our recent study, evaluating both surgical and nonsurgical cancer care, demonstrated that few differences in hospital characteristics, patient characteristics, or outcomes exist between PCHs and National Cancer Institute (NCI)-designated Cancer Centers. ${ }^{3}$

In this issue of Annals of Surgical Oncology, Mehta et al. ${ }^{7}$ report the results of their study evaluating outcomes of hepatic and pancreatic surgery at PCHs. They use textbook outcome (TO), a recently described composite outcome measure defined as the absence of complications, prolonged length of stay, mortality, or readmission within 90 days after discharge. Their study reports an increased likelihood of TO for patients who undergo hepatic or pancreatic surgery at a PCH compared with both NCIdesignated Cancer Centers and other non-exempt hospitals. Additionally, they report that TOs are associated with cost savings based on Medicare payments, and that for patients with a TO, payments were lower at a PCH. Their study concludes that PCHs may have improved outcomes and lower Medicare payments for hepatic and pancreatic procedures.

Although the study provided some evidence in favor of the PPS exemption policy, several limitations must be appreciated when the results are interpreted. First, by assessing only hepatic and pancreatic operations, this study evaluated a particularly complex subset of procedures. Although the study suggests that outcomes after these operations are improved at PCHs, this finding may not hold true for the treatment of other cancers that require less technically demanding procedures but affect significantly more patients. This is important to note given that the PPS exemption policy applies to all cancer patients and treatments, not only to patients undergoing complex surgical 
procedures. Moreover, the authors imply that the differences observed in their study are a result of the PPS exemption policy. However, it also is likely that PCHs, on the average, are simply higher-volume, experienced hepatic and pancreatic surgery centers. Similarly, there certainly are high-volume individual PPS hospitals that safely perform complex cancer surgery. This average association across many different hospitals for two procedures is not a justification of the policy.

Second, differences in payment incentives for PCHs and PPS hospitals may introduce bias into the coding of complications, and findings have shown that Medicare payment data have limited reliability. ${ }^{8}$ Specifically, PPS hospitals have financial incentives to capture complications such as urinary tract infection, acute renal failure, and sepsis because the codes for these complications influence reimbursement. However, PCHs may be less incentivized to code complications.

Third, variation in practice patterns and outcomes among the $11 \mathrm{PCHs}$ are unknown. Significant variation in case mix certainly exists, and it is therefore possible that the results of this study simply reflect differences observed for a small, highly specialized subset of PCHs rather than for the group as a whole.

Finally, the use of $\mathrm{TO}$ as an outcome measure in this study has important limitations. For patients undergoing operations known to have high rates of morbidity, it can be argued that a measure which focuses on a patient experiencing no complications whatsoever may be unrealistic. This approach weights all complications identically and therefore limits discrimination between patients who experience minor complications and those who experience life-threatening adverse events and does not assess how well these events are managed. Additionally, the use of an all-or-nothing outcome measure reduces the ability to specifically identify targets for quality improvement and does not capture patient-centered outcomes. Although these limitations do not invalidate the results of this study, they are important to consider when conclusions about the PPS exemption policy are drawn.

The increasing attention given to rising medical expenditures in Medicare and the health care system as a whole has brought the PPS exemption policy into question. Accumulating evidence suggests that the difference in average case complexity, which initially was the rationale for the PPS exemption policy, no longer exists. Additionally, by transitioning to a PPS-based compensation model, efficiency and value would be incentivized, and a large amount of public funds could potentially be saved. ${ }^{4}$ The study by Mehta et al. $^{7}$ provides evidence suggesting a different possible benefit of $\mathrm{PCHs}$ - that the care delivered by these centers may be better, and that a preferential payment scheme therefore might be justified.
Based on current evidence and the limitations of the study, however, the quality of care at $\mathrm{PCHs}$ cannot be reliably compared with that of hospitals governed by PPS, and such a conclusion cannot be drawn with certainty. Although PCHs unquestionably serve an important and vital role in advancing the science and quality of cancer care, additional transparency is required in the evaluation and continued inclusion of hospitals in this program. Because most cancer care is provided outside $\mathrm{PCHs}$, increased attention should be paid to cancer-specific quality measurement at all hospitals that manage patients with cancer. This will permit a broader evaluation and comparison of cancer care quality.

In conclusion, the study by Mehta et al. ${ }^{7}$ using a new outcome measure, textbook outcome, suggests that certain complex operations performed at PCHs may have better outcomes than those performed at NCI-designated cancer centers. Although this study offers a valuable and interesting perspective for the debate about the PPS exemption policy, the results alone do not justify the existence of this policy. As changes to the PPS exemption are considered in the future, an emphasis should be placed on increasing transparency regarding this program as well as periodic reviews of hospitals included as exempt and why if Medicare is to maintain this classification.

ACKNOWLEDGMENT Dr. Merkow is supported by the Agency for Healthcare Research and Quality (K12HS026385) and an Institutional Research Grant from the American Cancer Society (IRG-18163-24).

DISCLOSURE There are no conflicts of interest.

\section{REFERENCES}

1. Vanchieri C. Handful of cancer centers exempt from prospective payment system. J Natl Cancer Inst. 1991;83:907-8.

2. National Quality Forum. Performance measurement coordination strategy for PPS-exempt cancer hospitals-final report. Published June 2012. http://www.qualityforum.org/Publications/2012/06/M AP_Cancer_Hospitals_Final_Report.aspx. Accessed March 2020.

3. Merkow RP, Yang AD, Pavey E, et al. Comparison of hospitals affiliated with PPS-exempt cancer centers, other hospitals affiliated with NCI-designated cancer centers, and other hospitals that provide cancer care. JAMA Intern Med. 2019;179:1043-51.

4. U.S. Government Accountability Office. Medicare payment methods for certain cancer hospitals should be revised to promote efficiency (GAO-15-199). 2015. Available at https://www.gao.g ov/products/GAO-15-199. Accessed 5 April 2020.

5. Mehta R, Ejaz A, Hyer JM, et al. The impact of dedicated cancer centers on outcomes among Medicare beneficiaries undergoing liver and pancreatic cancer surgery. Ann Surg Oncol. 2019;26:4083-90.

6. Pfister DG, Rubin DM, Elkin EB, et al. Risk adjusting survival outcomes in hospitals that treat patients with cancer without information on cancer stage. JAMA Oncol. 2015;1:1303-10. 
7. Mehta R, Tsilimigras D, Paredes AZ, et al. Dedicated cancer centers are more likely to achieve a textbook outcome following hepatopancreatic surgery. Ann Surg Oncol. 2020. https://doi.org/ 10.1245/s10434-020-08279-y.

8. Riley GF. Administrative and claims records as sources of health care cost data. Med Care. 2009;47(7 Suppl 1):S51-5.
Publisher's Note Springer Nature remains neutral with regard to jurisdictional claims in published maps and institutional affiliations. 